\title{
Trade-Off between Collusion Resistance and User Life Cycle in Self-Healing Key Distributions with $t$-Revocation
}

\author{
Ratna Dutta \\ Claude Shannon Institute \\ NUI, Maynooth \\ Co. Kildare, IRELAND \\ ratna.dutta@nuim.ie
}

\author{
Sourav Mukhopadhyay \\ School of Electronic Engineering \\ Dublin City University \\ Dublin 9, IRELAND \\ msourav@eeng.dcu.ie
}

\author{
Tom Dowling \\ Claude Shannon Institute \\ NUI, Maynooth \\ Co. Kildare, IRELAND \\ tdowling@cs.nuim.ie
}

\begin{abstract}
We solve the problem of resisting the collusion attack in the one-way hash chain based self-healing key distributions introduced by Dutta et al., coupling it with the prearranged life cycle based approach of Tian et al. that uses the same self-healing mechanism introduced in Dutta et al. Highly efficient schemes are developed compared to the existing works with the trade-off in pre-arranged life cycles on users by the group manager and a slight increase in the storage overhead. For scalability of business it is often necessary to design more innovation and flexible business strategies in certain business models that allow contractual subscription or rental, such as subscription of mobile connection or TV channel for a pre-defined period. The subscribers are not allowed to revoke before their contract periods (life cycles) are over. Our schemes fit into such business environment. The proposed schemes are proven to be computationally secure and resist collusion between new joined users and revoked users together with forward and backward secrecy. The security proof is in an appropriate security model. Moreover, our schemes do not forbid revoked users from rejoining in later sessions unlike the existing selfhealing key distribution schemes.

Index Terms-session key distribution, self-healing, revocation, wireless networks, access structure, computational security, forward and backward secrecy.
\end{abstract}

\section{INTRODUCTION}

Efficient key distribution and key management over reliable channel has attracted much research interest and many classic and efficient schemes have been proposed [1], [4]. However these schemes are more appropriate for wired networks rather than wireless networks. Staddon et al. [15] introduced the selfhealing key distribution which addresses the problem of how to distribute session keys over an unreliable channel. Since then, self-healing key distribution has received much attention.

The central concept of self-healing key distribution schemes is that users, in a large and dynamic group communication over an unreliable network, can recover lost session keys on their own, even if they have lost some previous key distribution messages, without requesting additional transmissions from the group manager. This reduces network traffic and the risk of user exposure through traffic analysis and also decreases the work load on the group manager. The scheme is said to have $t$-revocation capability if the key distribution mechanism cannot be broken by any coalition of up to $t$ users. Selfhealing key distribution can find applications for many settings in wireless network, military oriented applications, rescue missions, scientific explorations, broadcast transmissions and various Internet services, where session keys are used only for short time periods.

Following the pioneering works by Staddon et al. [15], a number of self-healing key distribution approaches are proposed [15], [9], [2], [3], [7], [11], [12], [13] to achieve unconditional security in formal generalized model with improved efficiency. Further improvements in efficiency are obtained by relaxing the security slightly - from unconditional to computational [8], [5], [10]. The schemes [12], [13], [17] are based on vector space access structure instead of Shamir's [14] secret sharing. The hash chain based schemes [8], [5], [10] are computationally secure and are highly efficient compared to the existing unconditionally secure schemes. However, these hash chain based constructions have the fatal defect of not being collusion resistant in the sense that the collusion between new joined users and the revoked users are able to recover all the session keys which they are not entitled to. Among the collusion resistance self-healing key distribution schemes [2], [3], [12], [13], [16], [17], only [17] is hash chain based and uses the same self-healing mechanism as introduced in [5]. We address the problem of achieving collusion resistance for [5] following the approach of [17], with better efficiency gains in computation and storage. Similar to [17], we assume that a user can not choose the session for its revoked on its own. Rather the user is assigned a pre-arranged life cycle by the group manager and is forcefully revoked once its life cycle finishes. With this trade-off and a slight increase in the storage as compared to [5], we achieve the following:

(a) Our schemes resist collusion between the new joined users and the revoked users (unlike [5]), besides keeping forward and backward secrecy.

(b) Our schemes allow revoked users to join in later sessions with new identities, while this rejoining is prohibited for all the existing hash chain based self-healing key distribution schemes including [5] (except [17]).

(c) Storage and computation overheads in our scheme are less than in [17] as we do not use forward key chain. 
Self-healing key distribution is an ideal candidate to establish session keys in large and dynamic wireless networks in which session keys can be used only for a short time period due to frequent membership change. Thus assigning each user a pre-arranged life cycle by the group manager and not allowing the user to revoke before its life cycle completes, has natural appeal in many applications. Several innovative business models allow contractual subscription or rental by the service provider for the scalability of business and do not allow the user to revoke before his contract is terminated. Our schemes are suitable for such applications. Moreover, rejoining of revoked users can be done in our schemes at later session with new identities without compromising security, unlike the existing self-healing schemes.

\section{PRELIMINARIES}

\section{A. Key Distribution and Self-Healing}

Consider the following scenario for pay-per-view TV channel. Suppose $\left\{U_{1}, \ldots, U_{n}\right\}$ is a dynamically changing group of users (clients) and $\mathrm{GM} \notin\left\{U_{1}, \ldots, U_{n}\right\}$ is the group manager (the cable operator). The problem is how the GM can securely communicate with its dynamically changing group of clients over an insecure broadcast channel, so that only authorized clients (who pay) may view the content broadcast by the GM. The GM encrypts the content using a session key. We need a mechanism of distributing this session key in such a way that only the authorized users can recover this session key and decrypt the encrypted content. This mechanism is referred to as the key distribution problem. Our goal is to minimize the overhead for this key distribution keeping the following few issues in mind: $(a)$ group-rekeying is needed on each membership change; $(b)$ depending on specific nature of applications, we can adopt periodic group-rekeying; $(c)$ efficient and secure revocation as well as joining mechanisms are required for dynamic groups.

On top of this, $U_{i}$ may be off-line for some time due to power failure and may need to recover lost session keys immediately after going on-line again. The Self-healing property enables qualified users to recover lost session keys on their own, without requesting additional transmission from the GM.

The following notations are used throughout the paper.

$\begin{array}{ll}\mathcal{U} & : \text { set of all users in the networks } \\ U_{i} & : i \text {-th user } \\ \mathrm{GM} & : \text { group manager } \\ n & : \text { total number of users in the network } \\ m & : \text { total number of sessions } \\ t & : \text { the maximum number of compromised user } \\ F_{q} & : \text { a field of order } q \\ S_{i} & : \text { personal secret of user } U_{i} \\ \mathrm{SK}_{j} & : \text { session key generated by the GM in session } j \\ \mathcal{B}_{j} & : \text { broadcast message by the GM during session } j \\ Z_{i, j} & : \text { the information learned by } U_{i} \text { through } \mathcal{B}_{j} \text { and } S_{i} \\ R_{j} & : \text { the set of all revoked users in and before session } j \\ \mathcal{H} & : \text { a cryptographically secure one-way function } \\ S^{B} & : \text { backward key seed generated by the GM } \\ K_{i}^{B} & : i \text {-th backward key in the backward key chain }\end{array}$

\section{B. One-Way Functions}

Our constructions for self-healing key distribution are based on the practical intractability of one-way functions. Informally speaking, a one-way function $f: A \rightarrow B$ satisfies the following two properties where $A$ and $B$ are two finite sets: (a) $f$ is easy to compute; and $(b) f$ is hard to invert, i.e., it is difficult to get $x$ from $f(x)$. See [6] for a formal definition of one-way function.

\section{Our Security Model}

We now state the following definitions that are aimed to computational security for session key distribution adopting the security model of [9], [15].

Definition 2.1: (Session Key Distribution with privacy [15]) Let $i, t \in\{1, \ldots, n\}$ and $j \in\{1, \ldots, m\}$.

1) $\mathcal{D}$ is a session key distribution with privacy if

(a) for any user $U_{i}$, the session key $\mathrm{SK}_{j}$ is efficiently determined from $\mathcal{B}_{j}$ and $S_{i}$.

(b) for any set $R \subseteq \mathcal{U},|R| \leq t$, and $U_{i} \notin R$, it is computationally infeasible for users in $R$ to determine the personal key $S_{i}$.

(c) what users $U_{1}, \ldots, U_{n}$ learn from $\mathcal{B}_{j}$ cannot be determined from broadcasts or personal keys alone. i.e. if we consider separately either the set of $m$ broadcasts $\left\{\mathcal{B}_{1}, \ldots, \mathcal{B}_{m}\right\}$ or the set of $n$ personal keys $\left\{S_{1}, \ldots, S_{n}\right\}$, then it is computationally infeasible to compute session key $\mathrm{SK}_{j}$ (or other useful information) from either set.

2) $\mathcal{D}$ has $t$-revocation capability if given any $R \subseteq \mathcal{U}$, where $|R| \leq t$, the group manager GM can generate a broadcast $\mathcal{B}_{j}$, such that for all $U_{i} \notin R, U_{i}$ can efficiently recover the session key $\mathrm{SK}_{j}$, but the revoked users cannot. i.e. it is computationally infeasible to compute $\mathrm{SK}_{j}$ from $\mathcal{B}_{j}$ and $\left\{S_{l}\right\}_{U_{l} \in R}$.

3) $\mathcal{D}$ is self-healing if the following is true for any $j, 1 \leq$ $j_{1}<j<j_{2} \leq m$ :

(a) For any user $U_{i}$ who is a member in sessions $j_{1}$ and $j_{2}$, the key $\mathrm{SK}_{j}$ is efficiently determined by the set $\left\{Z_{i, j_{1}}, Z_{i, j_{2}}\right\}$.

(b) Let $1 \leq j_{1}<j<j_{2} \leq m$. For any disjoint subsets $L_{1}, L_{2} \subset \mathcal{U}$ where $L_{1}$ is a coalition of users removed before session $j_{1}$ and $L_{2}$ is a coalition of users joined from session $j_{2}$, and $\left|L_{1} \cup L_{2}\right| \leq t$, the set $\left\{Z_{l, j}\right\}_{U_{l} \in L_{1}, 1 \leq j \leq j_{1}} \cup$ $\left\{Z_{l, j}\right\}_{U_{l} \in L_{2}, j_{2} \leq j \leq m}$ cannot determine the session key $\mathrm{SK}_{j}$, $j_{1}<j<j_{2}$. i.e. $\mathrm{SK}_{j}$ can not be obtained by the coalition $L_{1} \cup L_{2}$.

Definition 2.2: ( $t$-wise forward and backward secrecy [9]) Let $t, i \in\{1, \ldots, n\}$ and $j \in\{1, \ldots, m\}$.

1) A key distribution scheme $\mathcal{D}$ guarantees $t$-wise forward secrecy if for any set $R \subseteq \mathcal{U}$, where $|R| \leq t$, and all $U_{l} \in R$ are revoked before session $j$, it is computationally infeasible for the members in $R$ together to get any information about $\mathrm{SK}_{j}$, even with the knowledge of group keys $\mathrm{SK}_{1}, \ldots, \mathrm{SK}_{j-1}$ before session $j$.

2) A session key distribution $\mathcal{D}$ guarantees $t$-wise backward secrecy if for any set $J \subseteq \mathcal{U}$, where $|J| \leq t$, and all $U_{l} \in J$ join after session $j$, it is computationally infeasible for the members in $J$ together to get any information about $\mathrm{SK}_{j}$, even with the knowledge of group keys $\mathrm{SK}_{j+1}, \ldots, \mathrm{SK}_{m}$ after session $j$. 


\section{Self-Healing Key Distributions With $t$-REVOCATION}

In this section, we present three efficient constructions for self-healing key distribution with $t$-revocation capability and refer them as SHKD -1 , SHKD -2 and SHKD -3 respectively. SHKD-1 uses Shamir's $(t, n)$-threshold secret sharing scheme For SHKD -2 and SHKD - 3, we adopt the same selfhealing technique and make use of a revocation polynomial for efficient revocation instead of using secret sharing schemes.

For all the following constructions, we consider a setting in which there is a group manager (GM) and $n$ users $\mathcal{U}=$ $\left\{U_{1}, \ldots, U_{n}\right\}$. All operations take place in a finite field, $F_{q}$, where $q$ is a large prime number $(q>n)$. In our setting, we allow a revoked user to rejoin the group in a later session. Let $\mathcal{H}: F_{q} \longrightarrow F_{q}$ be a cryptographically secure one-way function.

\section{A. Key Distribution SHKD - 1}

- Setup: Let $t$ be a positive integer. The group manager GM chooses independently and uniformly at random $m$ polynomials $f_{1}(x), \ldots, f_{m}(x) \in F_{q}[x]$, each of degree $t$. The GM randomly picks an initial backward key seed $S^{B} \in F_{q}$. It repeatedly applies (in the pre-processing time) the one-way function $\mathcal{H}$ on $S^{B}$ and computes the one-way backward key chain of length $m$ :

$$
K_{i}^{B}=\mathcal{H}\left(K_{i-1}^{B}\right)=\mathcal{H}^{i-1}\left(S^{B}\right) \text { for } 1 \leq i \leq m .
$$

The GM also selects at random $m$ numbers $\beta_{1}, \ldots, \beta_{m} \in F_{q}$. The $j$-th session key is computed as $\mathrm{SK}_{j}=\beta_{j}+K_{m-j+1}^{B}$.

Unlike the existing self-healing key distribution schemes, our setting allows a revoked user to rejoin the group in a later session with a new identity. However, we make the following restriction on the life cycle of each user as determined by the GM. Each user $U_{i}$ is first assigned a prearranged life cycle $\left(s_{i}, t_{i}\right)$, where $1 \leq s_{i}<t_{i} \leq m$, by the GM. i.e. $U_{i}$ is involved in $k_{i}=t_{i}-s_{i}+1$ many sessions and is not allowed to revoke before session $t_{i}$, however $U_{i}$ may get off-line during its life cycle due to power failure. Self-healing is needed at this point. Each user $U_{i}$, for $1 \leq i \leq n$, receives its personal secret keys corresponding to the $k_{i}$ sessions $S_{i}=\left\{f_{s_{i}}(i), \ldots, f_{t_{i}}(i) ; \beta_{s_{i}}, \ldots, \beta_{t_{i}}\right\}$ from the group manager via the secure communication channel between them.

- Broadcast: Let $R_{j}$ be the set of all revoked users for sessions in and before $j$ such that $\left|R_{j}\right| \leq t$ and $G_{j}$ be the set of all non-revoked users in session $j$. In the $j$-th session the GM first chooses a set of indices (different from 0 ) $W_{j}=$ $\left\{x_{1, j}, \ldots, x_{t, j}\right\}$ such that $I_{R_{j}} \subseteq W_{j}$, but $W_{j} \cap I_{G_{j}}=\emptyset$, where $I_{R_{j}}$ represents the indices of the users in $R_{j}, I_{G_{j}}$ denotes the set of indices of users in $G_{j}$ and $\emptyset$ is the empty set. The GM then computes $Z_{j}=K_{m-j+1}^{B}+f_{j}(0)$ and broadcasts the following message $\mathcal{B}_{j}$ :

$$
\mathcal{B}_{j}=\left\{x_{1, j}, \ldots, x_{t, j} ; f_{j}\left(x_{1, j}\right), \ldots, f_{j}\left(x_{t, j}\right) ; Z_{j}\right\} .
$$

- Session Key Recovery and Message Recovery: When a nonrevoked user $U_{i}$ receives the $j$-th session key distribution message $\mathcal{B}_{j}$, it interpolates $\left\{\left(x_{l, j}, f_{j}\left(x_{l, j}\right)\right\}_{l=1, \ldots, t}\right.$ and $\left(i, f_{j}(i)\right)$ to recover $f_{j}(0)$ by Lagrange's interpolation formula as follows:

$$
f_{j}(0)=\sum_{l=0}^{t} \Lambda_{l} f_{j}\left(x_{l, j}\right),
$$

where

$$
\Lambda_{l}=\prod_{\substack{k=0 \\ k \neq l}}^{t} \frac{-x_{k, j}}{x_{l, j}-x_{k, j}}
$$

with $x_{0, j}=i$. Then $U_{i}$ recovers the key $K_{m-j+1}^{B}$ as

$$
K_{m-j+1}^{B}=Z_{j}-f_{j}(0) .
$$

Finally, $U_{i}$ evaluates the current session key

$$
\mathrm{SK}_{j}=\beta_{j}+K_{m-j+1}^{B} .
$$

A user $U_{k}$ who either does not know its private information $\left(f_{j}(k) ; \beta_{j}\right)$ or who is a revoked user in $R_{j}$, i.e. $U_{k} \in W_{j} \cup R_{j}$, cannot compute $f_{j}(0)$ because $U_{k}$ knows insufficient number of points to interpolate the polynomial $f_{j}(x)$ from the broadcast message $\mathcal{B}_{j}$. Consequently, $U_{k}$ cannot recover the backward key $K_{m-j+1}^{B}$ and hence the $j$-th session key $\mathrm{SK}_{j}$.

- Add Group Members: When a new user wants to join the communication group starting from session $j$, the user gets in touch with the GM. The GM in turn picks an unused identity $v \in F_{q}$, assigns a life cycle $\left(s_{v}, t_{v}\right)$ to the new user with $s_{v}=j$, computes the personal secret keys corresponding to $k_{v}=t_{v}-s_{v}+1$ sessions $S_{v}=\left\{f_{s_{v}}(v), \ldots, f_{t_{v}}(v) ; \beta_{s_{v}}, \ldots, \beta_{t_{v}}\right\}$ and gives $S_{v}$ to this new group member via the secure communication channel between them.

\section{Complexity.}

- Storage overhead: Storage complexity of personal key for user $U_{i}$ with life cycle $\left(s_{i}, t_{i}\right)$ is $2\left(t_{i}-s_{i}+1\right) \log q$ bits.

- Communication overhead: Communication bandwidth for key management is $(t+1) \log q$ bits. Here we ignore the communication overhead for the broadcast of points $x_{l, j}$ for $l=1, \ldots, t$, as these identities can be picked from a small finite field.

- Computation overhead: The computation cost for key management is $2\left(t^{2}+t\right)$, which is essentially the number of multiplication operations needed to recover a $t$-degree polynomial by using Lagrange's interpolation formula.

\section{B. Key Distribution SHKD -2}

- Setup: The group manager randomly picks an initial backward key seed $S^{B} \in F_{q}$. It repeatedly applies (in the preprocessing time) the one-way function $\mathcal{H}$ on $S^{B}$ and computes the one-way key chain of length $m$ :

$$
K_{i}^{B}=\mathcal{H}\left(K_{i-1}^{B}\right)=\mathcal{H}^{i-1}\left(S^{B}\right)
$$

for $1 \leq i \leq m$. The $\mathrm{GM}$ also selects at random $m$ numbers $\beta_{1}, \ldots, \beta_{m} \in F_{q}$. The $j$-th session key is 
computed as $\mathrm{SK}_{j}=\beta_{j}+K_{m-j+1}^{B}$. The group manager chooses independently and uniformly at random $m t$-degree polynomials $f_{1}(x), \ldots, f_{m}(x) \in F_{q}[x], t<m, n$. Each user $U_{i}$ is first assigned a prearranged life cycle $\left(s_{i}, t_{i}\right)$, where $1 \leq s_{i}<t_{i} \leq m$, by the GM. Each user $U_{i}$, for $1 \leq i \leq n$, receives its personal secret keys corresponding to the $k_{i}=t_{i}-s_{i}+1$ sessions $S_{i}=\left\{f_{s_{i}}(i), \ldots, f_{t_{i}}(i) ; \beta_{s_{i}}, \ldots, \beta_{t_{i}}\right\}$ from the group manager via the secure communication channel between them.

- Broadcast: Let $R_{j}=\left\{U_{l_{1}}, \ldots, U_{l_{w_{j}}}\right\}$ be the set of all revoked users for sessions in and before $j$ such that $\left|R_{j}\right|=$ $w_{j} \leq t$. In the $j$-th session the group manager locates the backward key $K_{m-j+1}^{B}$ in the backward key chain and computes the polynomials

$$
\begin{gathered}
r_{j}(x)=\left(x-l_{1}\right) \cdots\left(x-l_{w_{j}}\right), \\
h_{j}(x)=K_{m-j+1}^{B} r_{j}(x)+f_{j}(x) .
\end{gathered}
$$

The polynomial $r_{j}(x)$ is called the revocation polynomial in session $j$ and the polynomial $f_{j}(x)$ plays the role of masking polynomial in session $j$. The group manager broadcasts the following message $\mathcal{B}_{j}$ :

$$
\mathcal{B}_{j}=R_{j} \cup\left\{h_{j}(x)\right\} .
$$

- Session Key Recovery: When a non-revoked user $U_{i}$ receives the $j$-th session key distribution message $\mathcal{B}_{j}$, it evaluates the polynomial $r_{j}(x)$ at point $i$ and recovers

$$
K_{m-j+1}^{B}=\frac{h_{j}(i)-f_{j}(i)}{r_{j}(i)} .
$$

Note that from the broadcast message $\mathcal{B}_{j}$, one gets $R_{j}$, thereby gets the indices of all revoked users, and consequently can easily compute the revocation polynomial. Finally, $U_{i}$ evaluates the current session key

$$
\mathrm{SK}_{j}=\beta_{j}+K_{m-j+1}^{B} .
$$

- Add Group Members: When a new user wants to join the communication group starting from session $j$, the user gets in touch with the GM. The GM in turn picks an unused identity $v \in F_{q}$, assigns a life cycle $\left(s_{v}, t_{v}\right)$ to the new user with $s_{v}=j$, computes the personal secret keys corresponding to $k_{v}=t_{v}-s_{v}+1$ sessions $S_{v}=\left\{f_{s_{v}}(v), \ldots, f_{t_{v}}(v) ; \beta_{s_{v}}, \ldots, \beta_{t_{v}}\right\}$ and gives $S_{v}$ to this new group member via the secure communication channel between them.

\section{Complexity.}

- Storage overhead: Storage complexity of personal key for user $U_{i}$ with life cycle $\left(s_{i}, t_{i}\right)$ is $2\left(t_{i}-s_{i}+1\right) \log q$ bits.

- Communication overhead: Communication bandwidth for key management is $(t+1) \log q$ bits. Here we ignore the communication overhead for the set of identities of revoked users, as these identities of revoked users can be picked from a small finite field.
- Computation overhead: The computation cost for key management is $2(t+1)$, which is the number of multiplication operations needed to find two points on two $t$-degree polynomials.

\section{Key Distribution SHKD -3}

- Setup: The group manager randomly picks the backward key seed $S^{B}$. It repeatedly applies (in the pre-processing time) the one-way hash function $\mathcal{H}$ on the initial backward key seed $S^{B}$ and computes the one-way key chain of length $m$ :

$$
K_{i}^{B}=\mathcal{H}\left(K_{i-1}^{B}\right)=\mathcal{H}^{i-1}\left(S^{B}\right)
$$

for $1 \leq i \leq m$. The $\mathrm{GM}$ also selects at random $m$ numbers $\beta_{1}, \ldots, \beta_{m} \in F_{q}$. The $j$-th session key is computed as $\mathrm{SK}_{j}=\beta_{j}+K_{m-j+1}^{B}$. The group manager chooses independently and uniformly at random a polynomial $f(x, y)=a_{0,0}+a_{1,0} x+a_{0,1} y+\cdots+a_{t, t} x^{t} y^{t}$ from $F_{q}[x, y]$, where $t$ is a positive integer, $t<m, n$. Each user $U_{i}$ is first assigned a prearranged life cycle $\left(s_{i}, t_{i}\right)$, where $1 \leq s_{i}<t_{i} \leq m$, by the GM. Each user $U_{i}$, for $1 \leq i \leq n$, receives its personal secret keys corresponding to the $k_{i}=t_{i}-s_{i}+1$ sessions $S_{i}=\left\{f(i, y) ; \beta_{s_{i}}, \ldots, \beta_{t_{i}}\right\}$ from the group manager via the secure communication channel between them.

- Broadcast: Let $R_{j}=\left\{U_{l_{1}}, \ldots, U_{l_{w_{j}}}\right\}$ be the set of all revoked users for sessions in and before $j$ such that $\left|R_{j}\right|=$ $w_{j} \leq t$. In the $j$-th session the group manager locates the forward key $K_{j}^{F}$ in the forward key chain and the backward key $K_{m-j+1}^{B}$ in the backward key chain and computes the polynomials

$$
\begin{gathered}
r_{j}(x)=\left(x-l_{1}\right) \cdots\left(x-l_{w_{j}}\right), \\
h_{j}(x)=K_{m-j+1}^{B} r_{j}(x)+f\left(x, \beta_{j}\right) .
\end{gathered}
$$

The polynomial $r_{j}(x)$ is called the revocation polynomial in session $j$ and the polynomial $f\left(x, \beta_{j}\right)$ plays the role of masking polynomial in session $j$. The group manager broadcasts the following message $\mathcal{B}_{j}$ :

$$
\mathcal{B}_{j}=R_{j} \cup\left\{h_{j}(x)\right\} .
$$

- Session Key Recovery: When a non-revoked user $U_{i}$ receives the $j$-th session key distribution message $\mathcal{B}_{j}$, it evaluates the polynomial $r_{j}(x)$ at point $i$, and recovers

$$
K_{m-j+1}^{B}=\frac{h_{j}(i)-f\left(i, \beta_{j}\right)}{r_{j}(i)} .
$$

Finally, $U_{i}$ computes the current session key

$$
\mathrm{SK}_{j}=\beta_{j}+K_{m-j+1}^{B} .
$$

Note that $r_{j}(x)$ is computable from the broadcast message $\mathcal{B}_{j}$ which carries the information of revoked uses' IDs and $\beta_{j}$ is transmitted to the non-revoked user $U_{i}$ during the Setup phase.

- Add Group Members: When a new user wants to join the communication group starting from session $j$, the user gets in touch with the GM. The GM in turn picks an 
unused identity $v \in F_{q}$, assigns a life cycle $\left(s_{v}, t_{v}\right)$ to the new user with $s_{v}=j$, computes the personal secret keys $S_{v}=\left\{f(v, y) ; \beta_{s_{v}}, \ldots, \beta_{t_{v}}\right\}$ and gives $S_{v}$ to this new group member via the secure communication channel between them.

\section{Complexity.}

- Storage overhead: Storage complexity of personal key for user $U_{i}$ with life cycle $\left(s_{i}, t_{i}\right)$ is $\left(t_{i}-s_{i}+t+2\right) \log q$ bits.

- Communication overhead: Communication bandwidth for key management is $(t+1) \log q$ bits. Here we ignore the communication overhead for the set of identities of revoked users, as these identities of revoked users can be picked from a small finite field.

-Computation overhead: The computation cost for key management is $2(t+1)$, which is the number of multiplication operations needed to find two points on two $t$-degree polynomials.

\section{Self-Healing}

We now explain our self-healing mechanism for the construction SHKD - 1. The self healing for the constructions SHKD -2 and SHKD -3 can be performed in a similar way. Let $U_{i}$ be a group member that receives session key distribution messages $\mathcal{B}_{j_{1}}$ and $\mathcal{B}_{j_{2}}$ in sessions $j_{1}$ and $j_{2}$ respectively, where $1 \leq j_{1} \leq j_{2}$, but not the session key distribution message $\mathcal{B}_{j}$ for session $j$, where $j_{1}<j<j_{2}$. User $U_{i}$ can still recover all the lost session keys $K_{j}$ for $j_{1}<j<j_{2}$ as desired by Definition $2.13(a)$ using the following steps.

(a) $U_{i}$ recovers from the broadcast message $\mathcal{B}_{j_{2}}$ in session $j_{2}$, the backward key $K_{m-j_{2}+1}^{B}$ and repeatedly apply the oneway function $\mathcal{H}$ on this and computes the backward keys $K_{m-j+1}^{B}$ for all $j, j_{1} \leq j<j_{2}$.

(b) $U_{i}$ then recovers all the session keys $\mathrm{SK}_{j}=\beta_{j}+$ $K_{m-j+1}^{B}$, for $j_{1} \leq j \leq j_{2}$.

Note that a user $U_{i}$ revoked in session $j$ cannot compute the backward keys $K_{m-j_{1}+1}^{B}$ for $j_{1}>j$. Moreover, since a user is not allowed to revoke before the end of its life cycle, $U_{i}$ revoked in $j$-th session means its life cycle completes at the $j$-th session. Consequently, $U_{i}$ does not have $\beta_{j_{1}}$ for $j_{1}$ J. As a result, revoked users cannot compute the subsequent session keys $\mathrm{SK}_{j_{1}}$ for $j_{1}>j$, as desired. This is forward secrecy.

Similarly, a user $U_{i}$ joined in session $j$ does not have $\beta_{j_{2}}$ for $j_{2}<j$, although it can compute the backward keys $K_{m-j_{2}+1}^{B}$ for $j_{2}<j$. This forbids $U_{i}$ to compute the previous session keys as desired. This is backward secrecy.

Now we will show that our construction can resist collusion required by Definition $2.13(b)$. Let $1 \leq j_{1}<j<j_{2} \leq m$. For any disjoint subsets $L_{1}, L_{2} \subset \mathcal{U}$, where $\left|L_{1} \cup L_{2}\right| \leq t$, no information about the session key $\mathrm{SK}_{j}, j_{1}<j<j_{2}$ can be obtained by the coalition $L_{1} \cup L_{2}$, where the set $L_{1}$ is a coalition of users removed before session $j_{1}$ and the set $L_{2}$ is a coalition of users joined from session $j_{2}$. Our constructions satisfy this property as illustrated below for the scheme SHKD -1 . The similar arguments hold for the other two constructions. Secret information held by users in $L_{1} \cup L_{2}$ and broadcasts in all the sessions do not get any information about $\mathrm{SK}_{j}$ for $j_{1} \leq j<j_{2}$. This is true because in the worst case, the coalition knows $S_{i}=\left\{f_{1}(i), \ldots, f_{j_{1}-1}(i) ; \beta_{1}, \ldots, \beta_{j_{1}-1}\right\}$ for $U_{i} \in L_{1}, S_{i}=\left\{f_{j_{2}}(i), \ldots, f_{m}(i) ; \beta_{j_{2}}, \ldots, \beta_{m}\right\}$ for $U_{i} \in L_{2}$, and $\mathcal{B}_{1}, \ldots, \mathcal{B}_{m}$. For each session $j, j_{1} \leq j<j_{2}$, the coalition can get backward key $K_{m-j+1}^{B}$ from $L_{2}$. However the session key $\mathrm{SK}_{j}$ is computed from the backward key $K_{m-j+1}^{B}$ and a random number $\beta_{j}$. The coalition $L_{1} \cup L_{2}$ cannot obtain the random numbers $\beta_{j}$ for $j_{1} \leq j<j_{2}$. Consequently, all the guess for $\mathrm{SK}_{j}$ with $j_{1} \leq j<j_{2}$ are equi-probable.

\section{SECURITY ANALYSIS}

The following theorems state that our constructions realize self-healing key distribution schemes with revocation capability. The proofs follow the argument in [5]. We omit the proofs here due to space constraints. They are available in the full version of the paper.

Theorem 4.1: Construction SHKD -1 is secure, selfhealing session key distribution scheme with privacy, $t$-revocation capability with respect to Definition 2.1 in our security model as described in Section D and achieves $t$-wise forward and backward secrecy with respect to Definition 2.2 in the model.

Theorem 4.2: Construction SHKD -2 is secure, selfhealing session key distribution scheme with privacy, $t$-revocation capability with respect to Definition 2.1 in the model and achieve $t$-wise forward and backward secrecy with respect to Definition 2.2 in the model.

Theorem 4.3: Construction SHKD-3 is secure, self-healing session key distribution scheme with privacy, $t$-revocation capability with respect to Definition 2.1 in the model and achieves $t$-wise forward and backward secrecy with respect to Definition 2.2 in the model.

\section{Performance Analysis}

Comparison of storage overhead, communication complexity and computation cost of each user (not the GM) in our constructions SHKD -1 , SHKD -2 , SHKD -3 with the existing self-healing session key distribution schemes is provided in Table I. In one hand our constructions reduce the communication complexity (bandwidth) to $O(t)$, whereas optimal communication complexity achieved by the previous schemes is $O(t j)$ at the $j$-th session. On the other hand, we achieve less computation cost. For a user $U_{i}$ at the $j$ th session, the computation cost is incurred by recovering all previous session keys upto the $j$-th session (worst case) by self-healing mechanism. The communication complexity and computation cost in our constructions do not increase as the number of session grows. These are the most prominent improvement of our schemes over the previous self-healing key distributions [2], [7], [9], [15].

Our constructions are based on [5] with the following subtle difference: 
TABLE I

COMPARISON AMONG DIFFERENT SELF-HEALING KEY DISTRIBUTION SCHEMES IN $j$-TH SESSION, $k_{i}=t_{i}-s_{i}+1$, WHERE $\left(s_{i}, t_{i}\right)$ IS THE LIFE CYCLE ASSIGNED TO USER $U_{i}$ BY THE GM

\begin{tabular}{|c|c|c|c|}
\hline Schemes & Storage Overhead & Communication Overhead & Computation Overhead \\
\hline Construction 3 of [15] & $(m-j+1)^{2} \log q$ & $\left(m t^{2}+2 m t+m+t\right) \log q$ & $2 m t^{2}+3 m t-t$ \\
Scheme 3 of [9] & $2(m-j+1) \log q$ & {$[(m+j+1) t+(m+1)] \log q$} & $m t+t+2 t j+j$ \\
Scheme 2 of [2] & $(m-j+1) \log q$ & $(2 t j+j) \log q$ & $2 j\left(t^{2}+t\right)$ \\
Construction 1 of [7] & $(m-j+1) \log q$ & $(t j+j-t-1) \log q$ & $2 t j+j$ \\
Construction 1 of [5] & $(m-j+1) \log q$ & $(t+1) \log q$ & $2 t+1$ \\
Construction 2 of [5] & $(m-j+1) \log q$ & $(t+1) \log q$ & $2\left(t^{2}+t\right)$ \\
SHKD - 1 & $2 k_{i} \log q$ & $(t+1) \log q$ & $2\left(t^{2}+t\right)$ \\
SHKD - 2 & $2 k_{i} \log q$ & $(t+1) \log q$ & $2 t+1$ \\
SHKD - 3 & $\left(k_{i}+t+1\right) \log q$ & $(t+1) \log q$ & $2 t+1$ \\
\hline
\end{tabular}

(a) No forward key chain is used.

(b) Each user $U_{i}$ is pre-assigned a life cycle $\left(s_{i}, t_{i}\right)$ by the GM following the work by [17]. This means user $U_{i}$ can participate in $k_{i}=t_{i}-s_{i}+1$ sessions and can not revoke before session $t_{i}$ is over.

(c) In contrast to [5], we have been able to resist collusion attack in our constructions by using pre-selected random numbers $\beta_{1}, \ldots, \beta_{m}$ (fixed) as part of users' secret keys apart from values on polynomials. A user $U_{i}$ with life cycle $\left(s_{i}, t_{i}\right)$ is given only $k_{i}=t_{i}-s_{i}+1$ values $f_{s_{i}}(i), \ldots, f_{t_{i}}(i)$ and the additional values $\beta_{s_{i}}, \ldots, \beta_{t_{i}}$ as part of its secret key by the GM via a secure communication channel between them at the initial setup. As compared to [5], we get increased storage for SHKD -1 and SHKD -2 if $k_{i}>\frac{m-j+1}{2}$, and for SHKD -3 if $k_{i}>m-j+1-(t+1)$. The communication and computation costs for all our schemes are the same as in [5].

(d) Revoked users may join at later sessions with new identities without violating any security.

We adapt the similar approach as [17] to achieve resistance to collusion attacks and the ability of revoked users to rejoin the group. However, in contrast to [17], we done away with forward hash key chains. Consequently, our schemes are more efficient than [17] in terms of both storage and computation cost.

\section{CONCLUSION}

We introduced three efficient self-healing key distribution schemes with $t$-revocation capability. Our proposed key distribution mechanism reduces storage, communication and computation costs over the previous approaches, and is scalable to very large groups in highly mobile, volatile and hostile wireless network. Our schemes are properly analyzed in an appropriate security model and are proven to be computationally secure and achieve both forward and backward secrecy. Also our schemes can resist collusion between the new joined user and the revoked users, with a trade-off in the storage overhead and assigning pre-determined life cycle to each user by the group manager. These trade-offs are often allowed in certain business models such as rental or subscription. Our setup therefore allows each user to choose its joining session at its will, but the session for its revocation is pre-selected by the group manager. However, unlike the existing self-healing key distribution schemes, rejoining of revoked users is permitted in our schemes at a later session with new identities.

\section{REFERENCES}

[1] S. Berkovit. How to Broadcast a Secret. Advances in Cryptology, Eurocrypt'91, LNCS 547, pp. 536-541, Springer-Verlag, 1991.

[2] C. Blundo, P. D'Arco, A. Santis, M. Listo. Design of Self-healing Key Distribution Schemes. Design Codes and Cryptology, N. 32, pp. 15-44, 2004.

[3] C. Blundo, P. D'Arco, A. Santis, M. Listo. Definitions and Bounds for Self-healing Key Distribution. Proceedings of the 31st ICALP 04, LNCS 3142, pp. 234-245, Springer-Verlag, 2004.

[4] R. Canetti, J. Garay, G. Itkis, D. Micciancio, M. Naor, B. Pinkas. Multicast Security: A Taxonomy and Some Efficient Constructions. In IEEE INFOCOMM'99, 1999.

[5] R. Dutta, E-C. Chang, S. Mukhopadhyay. Efficient Self-Healing Key Distributions with Revocation for Wireless Network using One Way Key Chains. Proceedings of the 5th ACNS 2007, LNCS, SpringerVerlag, 2007.

[6] O. Goldreich. Foundations of Cryptography: Basic Tools. Cambridge University Press, Cambridge, 2001.

[7] D. Hong, J. Kang. An Efficient Key Distribution Scheme with Selfhealing Property. IEEE Communication Letters'05, Vol. 9, pp. 759761, 2005.

[8] Y. Jiang, C. Lin, M. Shi and X. Shen. Self-healing Group Key Distribution with Time-limited Node Revocation for Wireless Sensor Networks. Ad Hoc Networks, Vol. 5, No. 1, pp. 14-23, 2007.

[9] D. Liu, P. Ning, K. Sun. Efficient Self-healing Key Distribution with Revocation Capability. Proceedings of the 10th ACM CCS'03, pp. 27-31, 2003.

[10] F. Kausar, S. Hassian, J. H. Park, and A. Masood. Secure Group Communication with Self-Healing and Rekeying in Wireless Sensor Networks. Proceedings of the Third International Conference, MSN 2007, pp. 737-748, 2007.

[11] S. More, M. Malkin, J. Staddon. Sliding-window Self-healing Key Distribution with Revocation. ACM Workshop on Survivable and Self-regenerative Systems'03, pp. 82-90, 2003.

[12] G. Saez. On Threshold Self-healing Key Distribution Schemes. Proceedings of Cryptography and Coding'04, LNCS 3796, pp. 340-354, Springer-Verlag, 2004.

[13] G. Saez. Self-healing Key Distribution Schemes with Sponsorization. IFIP International Federation for Information Processing'05, LNCS 3677, pp. 22-31, Springer-Verlag, 2005.

[14] A. Shamir. How to Share a Secret. Communications of the ACM, Vol. 22, No. 11, pp. 612-613, 1979.

[15] J. Staddon, S. Miner, M. Franklin, D. Balfanz, M. Malkin, D. Dean. Self-healing key distribution with Revocation. Proceedings of IEEE Symposium on Security and Privacy'02, pp. 224-240, 2002.

[16] B. Tian and M. He. Self-Healing Key Distribution Scheme with Novel Properties. International Journal of Network Security, Vol. 7, No. 2, pp. 147-152, 2008.

[17] B. Tian, S. Han, T-S Dillon, and S. Das. A Self-Healing Key Distribution Scheme Based on Vector Space Secret Sharing and One Way Hash Chains. Proceedings of IEEE WoWMoM 2008. 